\title{
M - STAR - Irresolute Topological Vector Spaces
}

\author{
Raja Mohammad Latif \\ Department of Mathematics and Natural Sciences \\ College of Sciences and Humanities \\ Prince Mohammad Bin Fahd University \\ Al - Khobar 31952 Saudi Arabia
}

\begin{abstract}
In 2016 A. Devika and A. Thilagavathi introduced a new class of sets called $\mathrm{M}^{*}$ - open sets and investigated some properties of these sets in topological spaces. In this paper, we introduce and study a new class of spaces, namely $\mathrm{M}^{*}$-irresolute topological vector spaces via $M^{*}$ - open sets. We explore and investigate several properties and characterizations of this new notion of $\mathrm{M}^{*}$-irresolute topological vector space. We give several characterizations of $\mathrm{M}^{*}$ - Hausdorff space. Moreover, we show that the extreme point of the convex subset of $\mathrm{M}^{*}$-irresolute topological vector space $\mathrm{X}$ lies on the boundary.
\end{abstract}

Keywords-Topological space, $\mathrm{M}^{*}$-open set, $M^{*}$ - closed set, $M^{*}$-irresolute topological vector space, $\mathrm{M}^{*}$-irresolute function.

\section{INTRODUCTION}

The concept of topological vector spaces was first given by Kolmogroff in 1934. Since then, different researchers have explored many interesting and useful properties of topological vector spaces. Due to nice properties, topological vector spaces earn a great importance and remain a topological notion in various advanced branches of mathematics like fixed point theory, operator theory, rational inequalities, etc. Now a days the researchers not only make use of topological vector spaces in other fields for developing new notions but also extend the concept of topological vector spaces with every possible way, making this field of study a more convenient and understandable. Recently, M. Khan et al. (2015) introduced the $s$-topological vector spaces, a generalization of topological vector spaces. In 2016, Khan and Iqbal introduced the irresolute topological vector spaces which are particular brands of $s$-topological vector spaces, but irresolute topological vector spaces are independent of topological vector spaces. Ibrahim (2017) initiated the study of $\alpha$-topological vector spaces. Ram et al. (2018) introduced the notion of almost s-topological vector spaces. Recently in 2016 A. Devika and A. Thilagavathi introduced a new class of open sets called $\mathrm{M}^{*}$-open sets in topological spaces and showed that the family of all $\mathbf{M}^{*}$-open subsets of a topological space $(X, \tau)$ forms a topology on $X$. We introduce $\mathrm{M}^{*}$-irresolute topological vector spaces by using $\mathrm{M}^{*}$-open sets and investigate several properties and characterizations of this notion of $\mathrm{M}^{*}$-irresolute topological vector space.

\section{M"-OPEN SETS IN TOPOLOGICAL}

\section{SPACES}

The $(X, \tau)$ and $(Y, \sigma)$ (or simply, $X$ and $Y$ ) denote topological spaces on which no separation axioms are assumed unless explicitly stated. A subset $A$ of a topological space $(X, \tau)$ is said to be open if $A \in \tau$. A subset $A$ of a topological space $X$ is said to be closed if the set $X \mathbf{B} A$ is open. The interior of a subset $A$ of a topological space $X$ is defined as the union of all open sets contained in $A$. It is denoted by $\operatorname{Int}(A)$. The closure of a subset $A$ of a topological space $X$ is defined as the intersection of all closed sets containing $A$. 
We start recalling following definitions and results from [Devika \& Thilavagathi:2016], which are necessary for this study in sequel.

Definition 2.1. A subset $A$ of a topological space $(X, \tau)$ is said to be semi-open set if $A \subseteq C l\lfloor\operatorname{Int}(A)\rfloor$.

Definition 2.2. A subset $A$ of a topological space $(X, \tau)$ is said to be regular open set if $A=\operatorname{Int}\lfloor C l(A)\rfloor$ and a regular closed set if $A=C l\lfloor\operatorname{Int}(A)\rfloor$.

Definition 2.3. Let $(X, \tau)$ be a topological space and $A \subseteq X$. A point $x \in X$ is said to be $\theta$-interior point of $A$ if there exists an open set $U$ containg $x$ such that $U \subseteq C l(U) \subseteq A$. The set of all $\theta$-interior points of $A$ is said to be the $\theta$-interior of $A$ and is denoted by $\operatorname{Int}_{\theta}(A)$. A subset $A$ of $X$ is said to be $\theta$-open if $A=\operatorname{Int}_{\theta}(A)$. The set of all $\theta$-open sets in a topological space $(X, \tau)$ is denoted by $\tau_{\theta}$. We note that $\tau_{\theta}$ is a topology on $X$ and $\tau_{\theta} \subseteq \tau$. For a subset $A$ of $X$, $\theta$-closure of $A$ denoted by $C l_{\theta}(A)$ is defined by

$$
C l_{\theta}(A)=
$$

$\bigcap\{B \subseteq X: A \subseteq B$ and $B$ is $\theta$-closed in $X\}$.

Definition 2.4. A point $x \in X$ is called the $\delta$-cluster point of $A \subseteq X$ if $A \cap \operatorname{Int}\lfloor C l(U)\rfloor \neq \phi$ for every open set $U$ of $X$ containing $x$. The set of all $\delta$-cluster points of $A$ is called the $\delta$-closure of $A$, denoted by $C l_{\delta}(A)$. A subset $A \subseteq X$ is called $\delta$-closed if $A=C l_{\delta}(A)$. The complement of a $\delta$-closed set is called $\delta$-open. The collection of all $\delta$-open sets in a topological space $(X, \tau)$ forms a topology $\tau_{\delta}$ on $X$, called the semigeneralization topology of $\tau$, weaker than $\tau$ and the class of all regular open sets in $\tau$ forms an open basis for $\tau_{\delta}$.

We observe that for any topological space $(X, \tau)$ the relation $\tau_{\theta} \subseteq \tau_{\delta} \subseteq \tau$ always holds.
Definition 2.5. A subset $A$ of a topological space $(X, \tau)$ is said to be $\theta$-semi-open set if $A \subseteq C l\left\lfloor\operatorname{Int}_{\theta}(A)\right\rfloor$.

Definition 2.6. A subset $A$ of a topological space $(X, \tau)$ is said to be $M$-open set if $A \subseteq C l\left\lfloor\operatorname{Int}_{\theta}(A)\right\rfloor \cup \operatorname{Int}\left\lfloor C l_{\delta}(A)\right\rfloor$.

Definition 2.7. The complement of a semi-open (resp. $\theta$-semi-open, $M$-open) set is called semi-closed (resp. $\theta$-semi-closed, $M$-closed).

Definition 2.8. The intersection of all semi-closed

(resp. $\theta$-semi-closed, $M$-closed) sets containing $A$ is called the semi-closure (resp. $\theta$-semi-closure, $M$-closure) of $A$ and is denoted by $\operatorname{scl}(A)$ (resp. $s \mathrm{Cl}_{\theta}(A), M-C l(A)$ ).

Definition 2.9. The union of all semi-open (resp. $\theta$-semi-open, $M$-open) sets containing $A$ is called the semi-interior (resp. $\theta$-semi-interior, $M$-interior) of $A$ and is denoted by $\operatorname{sint}(A)$ (resp. $\left.\operatorname{sint}_{\theta}(A), M-\operatorname{Int}(A)\right)$.

Lemma 2.10. Let $(X, \tau)$ be a topological space and $A, B \subseteq X$. Then the following statements are true :

(1) $A$ is $\theta$-openopen if and only if $A=\operatorname{Int}_{\theta}(A)$.

(2) $\operatorname{Int}_{\theta}(A)$ is the union of all $\theta$-open sets of $X$ whose closures are contained in $A$.

(3) For any subset $A$ of $\quad X$,
$A \subseteq C l(A) \subseteq C l_{\delta}(A) \subseteq C l_{\theta}(A)$
$\left(r e s p . \operatorname{Int}_{\theta}(A) \subseteq \operatorname{Int}_{\delta}(A) \subseteq \operatorname{Int}(A) \subseteq A\right)$.
$\operatorname{Int}_{\theta}(A \cap B)=\operatorname{Int}_{\theta}(A) \cap \operatorname{Int}_{\theta}(B)$.
$\operatorname{Int}_{\theta}(A) \cup \operatorname{Int}_{\theta}(B) \subseteq \operatorname{Int}_{\theta}(A \cup B)$.
(5) $C l_{\theta}(A \cap B)=C l_{\theta}(A) \cap C l_{\theta}(B)$.
$C l_{\theta}(A \cup B)=C l_{\theta}(A) \cup C l_{\theta}(B)$.


Definition 2.11. Let $(X, \tau)$ be a topological space. Then a subset $A$ of a space $(X, \tau)$ is said to be,

(1) an $M^{*}$-open set if $A \subseteq \operatorname{Int}\left\lfloor C l\left(\operatorname{Int}_{\theta}(A)\right)\right\rfloor$. an $M^{*}$-closed set if $C l\left\lfloor\operatorname{Int}\left(C l_{\theta}(A)\right)\right\rfloor \subseteq A$.

Lemma 2.12. Let $(X, \tau)$ be a topological space. Then the following statements are true :

(1). Every $\theta$-open set is an $M^{*}$ - open set.

(2) Every $M^{*}$-open set is a $\theta$-semi-open set.

(3) Every $M^{*}$-open set is an $M$-open set.

Lemma 2.13. Let $(X, \tau)$ be a topological space. Then the following statements are true:

(1) The arbitrary union of $M^{*}$-open sets is $M^{*}$-open.

(2) The arbitrary intersection of $M^{*}$-closed sets is $M^{*}$-closed.

Lemma 2.14. For a topological space $(X, \tau)$ the family of all $M^{*}$-open sets of $X$ forms a topology denoted by $\tau_{M^{*}}=M^{*}-O(X, \tau)=M^{*}-O(X)$ for $X$.

Definition 2.15. Let $A$ be a subset of a space $(X, \tau)$. Then,

(1) The intersection of all $M^{*}-$ closed sets containing $A$ is called the $M^{*}$-closure of $A$ and is denoted by $M^{*}-C l(A)$.

(2) The union of all $M^{*}$-open sets contained in $A$ is called the $M^{*}$-Interior of $A$ and is denoted by $M^{*}-\operatorname{Int}(A)$.

Theorem 2.16. The following statements hold for a subset $A$ of a space $(X, \tau)$.

(1) $A$ is $M^{*}$-open if and only if $A=A \cap \operatorname{Int}\left\lfloor C l\left(\operatorname{Int}_{\theta}(A)\right)\right\rfloor$.
(2) $A$ is $M^{*}$-closed if and only if $A=A \cup C l\left\lfloor\operatorname{Int}\left(C l_{\theta}(A)\right)\right\rfloor$.

Theorem 2.17. The following statements hold for a subset $A$ of a space $(X, \tau)$.

(1) $M^{*}-\operatorname{Int}(A)=A \bigcap \operatorname{Int}\left|\operatorname{Cl}\left(\operatorname{Int}_{\theta}(A)\right)\right|$.

(2) $M^{*}-C l(A)=A \cup C l\left[\operatorname{Int}\left(C l_{\theta}(A)\right)\right]$.

Theorem 2.18. Let $A$ be a subset of a topological space $(X, \tau)$. Then the following statements hold :

(1) $A$ is an $M^{*}$-open set if and only if $A=M^{*}-\operatorname{Int}(A)$.

(2) $A$ is an $M^{*}$-closed set if and only if $A=M^{*}-C l(A)$.

Prpoosition 2.19. A subset $A$ of a topological space $(X, \tau)$ is an $M^{*}$-closed set if and only if $X \mathbf{B} A$ is an $M^{*}$-open set. The set of all $M^{*}-$ closed sets in $(X, \tau)$ is denoted by $M^{*}-C(X)=M^{*}-C(X, \tau)$.

Theorem 2.20 Let $A$ be a subset of a topological space $(X, \tau)$. Then the following statements hold :

(1) $M^{*}-C l(X \mathbf{B} A)=X \mathbf{B} M^{*}-\operatorname{Int}(A)$.

(2) $M^{*}-\operatorname{Int}(X \mathbf{B} A)=X \mathbf{B} M^{*}-C l(A)$.

(3) If $A \subseteq B$ then $M^{*}-C l(A) \subseteq M^{*}-C l(B)$ and $M^{*}-\operatorname{Int}(A) \subseteq M^{*}-\operatorname{Int}(B)$.

(4) $M^{*}-C l\left[M^{*}-C l(A)\right]=M^{*}-C l(A) \quad$ and $M^{*}-\operatorname{Int}\left\lfloor M^{*}-\operatorname{Int}(A)\right\rfloor=M^{*}-\operatorname{Int}(A)$.

(5) $\quad M^{*}-C l(A) \cup M^{*}-C l(B) \subseteq M^{*}-C l(A \cup B)$ and

$M^{*}-\operatorname{Int}(A) \cup M^{*}-\operatorname{Int}(B) \subseteq M^{*}-\operatorname{Int}(A \cup B)$.

(6) $M^{*}-C l(A \cap B) \subseteq M^{*}-C l(A) \cap M^{*}-C l(B)$

and

$M^{*}-\operatorname{Int}(A \cap B) \subseteq M^{*}-\operatorname{Int}(A) \cap M^{*}-\operatorname{Int}(B)$.

(7) $M^{*}-C l(A)=A \bigcup \operatorname{sint}_{\theta}\left[C l_{\theta}(A)\right]$.

(8) $M^{*}-\operatorname{Int}(A)=A \bigcap s C l_{\theta}\left[\operatorname{Int}_{\theta}(A)\right]$. 
Theorem 2.21. The following are equivalent for a subset $A$ of $(X, \tau)$.

(1) $A$ is an $M^{*}$-open set.

(2) $A \subseteq s C l_{\theta}\left\lfloor\operatorname{Int}_{\theta}(A)\right\rfloor$.

(3) $s C l_{\theta}(A)=s C l_{\theta}\left\lfloor\operatorname{Int}_{\theta}(A)\right\rfloor$.

Theorem 2.22. Let $A$ be a subset of a space $(X, \tau)$. Then the following are equivalent :

(1) $A$ is an $M^{*}-$ closed set.

(2) $\operatorname{sint}_{\theta}\left\lfloor C l_{\theta}(A)\right\rfloor \subseteq A$.

(3) $\operatorname{sint}_{\theta}(A)=\operatorname{sint}_{\theta}\left\lfloor C l_{\theta}(A)\right\rfloor$.

Definition 2.23. A subset $A$ of a topological space $(X, \tau)$ is said to be an $M^{*}$-neighborhood (Briefly $\left.M^{*}-n b d\right)$ of a point $p \in X$ if there exists an $M^{*}$-open set $W$ such that $p \in W \subseteq A$. The class of $M^{*}-$ neighborhoods of $p \in X$ is called the $M^{*}$-neighborhood system of $p$ and denoted by $M^{*}-N_{p}(X)=M^{*}-N_{p}(X, \tau)=M^{*}-N(p)$.

Theorem 2.24. A subset $G$ of a space $X$ is $M^{*}$-open if and only if it is $M^{*}-n b d$ for every point $p \in G$.

Definition 2.25 A mapping $f:(X, \tau) \rightarrow(Y, \sigma)$ is called $M^{*}$-irresolute at a point $x \in X$ if for all $M^{*}$-open subsets $V$ in $Y$ containing $f(x)$, there is an $M^{*}$-open subset $U$ of $X$ such that $f(U)$ is a subset of $V$. The function $f$ will be called $M^{*}$-irresolute if $f$ is $M^{*}$-irresolute at each point $x \in X$.

Theorem 2.26 Let $f:(X, \tau) \rightarrow(Y, \sigma)$ be a function. Then the following statements are equivalent.

(1) $f$ is $M^{*}$-irresolute.

(2) For each $x \in X$ and each $M^{*}$-neighborhood $V$ of $f(x)$ in $Y$, there is an $M^{*}$-neighborhood $U$ of $x$ in $X$ such that $f(U) \subseteq V$.
(3) The inverse image of every $M^{*}$-closed subset of $Y$ is an $M^{*}$-closed subset of $X$.

(4) The inverse image of every $M^{*}$-open subset of $Y$ is an $M^{*}$-open subset of $X$.

Definition 2.27. A function $f:(X, \tau) \rightarrow(Y, \sigma)$ is called $M^{*}$-continuous if $f^{-1}(V)$ is $M^{*}$-open set in $X$ for every open set $V$ in $Y$. Proposition 2.28. A function $f:(X, \tau) \rightarrow(Y, \sigma)$ is called $M^{*}$-continuous if and only if $f^{-1}(V)$ is $M^{*}$-closed set in $X$ for every closed set $V$ in $Y$.

Definition 2.29. A function $f:(X, \tau) \rightarrow(Y, \sigma)$ is called Pre- $M^{*}$-open if and only if the image set $f(U)$ is $M^{*}$-open set in $Y$ for every $M^{*}$-open set $U$ in $X$.

Proposition 2.30. A bijection function $f:(X, \tau) \rightarrow(Y, \sigma)$ is called $M^{*}$-homeomorphism if $f$ is pre-M*-open and $M^{*}$-irreseolute.

\section{PROPERTIES OF M*-IRRESOLUTE TOPOLOGICAL VECTOR SPACES}

In this section, we define and investigate some basic properties of $M^{*}$-irresolute topological vector spaces.

Definition 3.1. A topological space $\left(X_{(K)}, \tau\right)$ is called an $M^{*}$-irresolute topological vector space $\left(M^{*} I T V S\right)$ whenever the following conditions are satisfied.

(1) for each $x, y \in X$ and for each $M^{*}$-open neighborhood $W$ of $x+y$ in $X$, there exist $M^{*}$-open neighborhoods $U$ and $V$ in $X$ of $x$ and $y$ respectively, such that $U+V \subseteq W$.

(2) for each $x \in X, \lambda \in K$ and for each $M^{*}$-open neighborhood $W$ of $\lambda x$ in $X$, there exist $M^{*}$-open neighborhoods $U$ of $\lambda$ in $K$ and $V$ of $x$ in $X$, such that $U . V \subseteq W$.

Theorem 3.2. Let $\left(X_{(K)}, \tau\right)$ be an $M^{*}$-irresolute topological vector space. Then the following 
assertions are true:

(1) The (left) right translation mapping $T_{x}: X \rightarrow X$ defined by $T_{x}(y)=y+x$; for all $x, y \in X$, is $M^{*}$-irresolute.

(2) The multiplication mapping $M_{\lambda}: X \rightarrow X$, defined by $M_{\lambda}(x)=\lambda x$, for all $x \in X$, is $M^{*}$-irresolute.

Proof. (1) Let $W$ be an $M^{*}$-open neighborhood of $T_{x}(y)=x+y$. Then by definition, there exist $M^{*}$-open neighborhoods $U$ and $V$ in $X$ containing $y$ and $x$ respectively, such that $U+V \subseteq W . \quad$ This gives that $T_{x}(U)=U+x \subseteq U+V \subseteq W$. This proves that, $T_{x}: X \rightarrow X$ is an $M^{*}$-irresolute mapping.

(2) Let $x \in X, \lambda \in K$. Then $M_{\lambda}(x)=\lambda x$. Let $W$ be any $M^{*}$-open neighborhood $W$ of $\lambda x$. Then by definition of $M^{*} I T V S$, there exist $M^{*}$-open neighborhoods $U$ in $K$ of $\lambda$ and $V$ in $X$ of $x$, such that $U . V \subseteq W$. This gives that $M_{\lambda}(V)=\lambda V \subseteq U . V \subseteq W$. This proves that $M_{\lambda}: X \rightarrow X$ is $M^{*}$-irresolute mapping.

Theorem 3.3. Let $\left(X_{(K)}, \tau\right)$ be an $M^{*}$-irresolute topological vector space. Let $A \in M^{*}-O(X)$. Then the following statements are true.

(1) $x+A \in M^{*}-O(X)$, for every $x \in X$.

(2) $\lambda A \in M^{*}-O(X)$, for every non-zero scalar $\lambda \in K$.

Proof. (1) $y \in x+A$. Then $y=x+a$ for some $a \in A$. By definition of $M^{*}$-irresolute topological vector spaces, there exist $M^{*}$-open sets $U$ and $V$ in $X$ containing $-x$ and $y$ respectively such that $U+V \subseteq A$. This gives $-x+V \subseteq U+V \subseteq A$. This implies $y \in V \subseteq x+A$. Therefore $y \in M^{*}-\operatorname{Int}(x+A)$. Hence, $x+A=M^{*}-\operatorname{Int}(x+A)$. This proves that $x+A$ is $M^{*}$-open in $X$.

(2) Let $x \in \lambda A$. Then $x=\lambda a$ for some $a \in A$. Thus $a=\frac{1}{\lambda} x \in A$. By definition of $M^{*}$-irresolute topological vector spaces, there exist $M^{*}$-open sets
$U$ in $K$ containing $\frac{1}{\lambda}$ and $V$ in $X$ containing $x$ such that $U . V \subseteq A$. This implies that $a=\frac{1}{\lambda} x \in \frac{1}{\lambda} V \subseteq U . V \subseteq A$. Hence $x \in V \subseteq \lambda A$. Thus we obtain $x \in M^{*}-\operatorname{Int}(\lambda A)$. Therefore, it follows that $\lambda A \subseteq M^{*}-\operatorname{Int}(\lambda A)$. Hence, $\lambda A=M^{*}-\operatorname{Int}(\lambda A)$. This shows that $\lambda A \in M^{*}-O(X)$.

Corollary 3.4. Let $\left(X_{(K)}, \tau\right)$ be an $M^{*}$-irresolute topological vector space. Let $A$ be an $M^{*}$-open subset of $X$. Then the following statements are true. (1) $x+A \subseteq M^{*}-C l\left[M^{*}-\operatorname{Int}(x+A)\right]$ for each $x \in X$.

(2) $\lambda A \subseteq M^{*}-C l\left[M^{*}-\operatorname{Int}(\lambda A)\right]$ for any non-zero scalar $\lambda$.

Theorem 3.5. Let $\left(X_{(K)}, \tau\right)$ be an $M^{*}$-irresolute topological vector space. Let $A \in M^{*}-O(X)$ and $B$ be any subset of $X$. Then prove that $A+B \in M^{*}-O(X)$.

Proof. Suppose $A \in M^{*}-O(X)$. and $B \subseteq X$. Then, for each $b \in B$ and by Theorem 3.3 (1), we have $A+b \in M^{*}-O(X)$ Then $A+B=\bigcup\{A+b: b \in B\}$ is a union of $M^{*}$-open sets. Since arbitrary union of $M^{*}$-open sets is $M^{*}$-open, therefore $A+B$ is $M^{*}$-open in $X$.

Corollary 3.6. Suppose $\left(X_{(K)}, \tau\right)$ is $M^{*}$-irresolute topological vector space and let $A \in M^{*}-O(X)$. Then the set $U=\bigcup_{n=1}^{\infty}(n A)$ is $M^{*}$-open in $X$.

Theorem 3.7. Let $\left(X_{(K)}, \tau\right)$ be an $M^{*}$-irresolute topological vector space. Let $A \subseteq X$. Then the following statements are true

(1) $M^{*}-\operatorname{Int}(x+A)=x+M^{*}-\operatorname{Int}(A)$ for any $x \in X$.

(2) $M^{*}-\operatorname{Int}(\lambda A)=\lambda\left[M^{*}-\operatorname{Int}(A)\right]$ for non-zero scalar $\lambda \in K$.

Proof. (1) By Theorem $3.3(1), x+M^{*}-\operatorname{Int}(A)$ is $M^{*}$-open. Therefore $x+M^{*}-\operatorname{Int}(A) \subseteq x+A$ implies $x+M^{*}-\operatorname{Int}(A) \subseteq M^{*}-\operatorname{Int}(x+A) . \quad$ Now let $z \in M^{*}-\operatorname{Int}(x+A)$. Then $z=x+y$ for some 
$y \in A$. By definition of $M^{*} I T V S$, there exist $M^{*}$-open sets $U$ and $V$ in $X$ containing $x$ and $y$ respectively, such that $U+V \subseteq x+M^{*}-\operatorname{Int}(A)$.

This gives that $z=x+y \in x+V \subseteq U+V \subseteq x+M^{*}-\operatorname{Int}(A)$.

Therefore it follows that $M^{*}-\operatorname{Int}(x+A) \subseteq x+M^{*}-\operatorname{Int}(A)$.

(2) By Theorem 3.3(2), $\lambda\left[M^{*}-\operatorname{Int}(A)\right]$ is $M^{*}$-open. Thus $\lambda\left[M^{*}-\operatorname{Int}(A)\right] \subseteq \lambda A$ implies that $\lambda\left[M^{*}-\operatorname{Int}(A)\right] \subseteq M^{*}-\operatorname{Int}(\lambda A) . \quad$ Next, $\quad$ if $y \in M^{*}-\operatorname{Int}(\lambda A)$, then $y=\lambda x$ for some $x \in A$. By definition of $M^{*} I T V S$, there exist $M^{*}$-open sets $U$ of $\lambda$ in $K$ and $V$ of $x$ in $X$ such that $U . V \subseteq M^{*}-\operatorname{Int}(\lambda A) . \quad$ Therefore, $y=\lambda x \in \lambda V \subseteq U . V \subseteq M^{*}-\operatorname{Int}(\lambda A) \subseteq \lambda A . \quad$ This implies that $x \in V \subseteq A$. Since $V$ is $M^{*}$-open. Thus $x \in M^{*}-\operatorname{Int}(A)$. Consequently, $y=\lambda x \in \lambda\left[M^{*}-\operatorname{Int}(A)\right]$. Hence we conclude that $M^{*}-\operatorname{Int}(\lambda A)=\lambda\left[M^{*}-\operatorname{Int}(A)\right]$.

Theorem 3.8. Let $\left(X_{(K)}, \tau\right)$ be an $M^{*}$-irresolute topological vector space. Let $A, B \subseteq X$. Then $M^{*}-\operatorname{Int}(A)+M^{*}-\operatorname{Int}(B) \subseteq M^{*}-\operatorname{Int}(A+B)$.

Proof. We know that $M^{*}-\operatorname{Int}(A) \subseteq A$ and $M^{*}-\operatorname{Int}(B) \subseteq B$. Thus

$M^{*}-\operatorname{Int}(A)+M^{*}-\operatorname{Int}(B) \subseteq A+B$. By Theorem 3.5, $M^{*}-\operatorname{Int}(A)+M^{*}-\operatorname{Int}(B)$ is $M^{*}$-open. Therefore we have $\quad M^{*}-\operatorname{Int}(A)+M^{*}-\operatorname{Int}(B)=$ $M^{*}-\operatorname{Int}\left[M^{*}-\operatorname{Int}(A)+M^{*}-\operatorname{Int}(B)\right] \subseteq M^{*}-\operatorname{Int}(A+B)$. Hence $M^{*}-\operatorname{Int}(A)+M^{*}-\operatorname{Int}(B) \subseteq M^{*}-\operatorname{Int}(A+B)$.

Theorem 3.9. Let $F$ be any $M^{*}$-closed subset of an $M^{*}$-irresolute topological vector space $X$. Then the following statements are true:

(1) $x+F \in M^{*}-C(X)$, for every $x \in X$.

(2) $\lambda F \in M^{*}-C(X)$, for each non - zero scalar $\lambda \in K$.

Proof. (1) Suppose that $y \in M^{*}-C l(x+F)$. Consider $z=-x+y$ and let $W$ be any $M^{*}$-open set in $X$ containing $z$. Then there exist $M^{*}$-open sets $U$ and $V$ in $X$ such that $-x \in U, y \in V$ and $U+V \subseteq W . \quad$ Since $\quad y \in M^{*}-C l(x+F)$, $(x+F) \cap V \neq \phi$. So, there is an element $a \in(x+F) \cap V$. Thus $a \in x+F$ and $a \in V$. Hence $-x+a \in F \quad$ and $\quad-x+a \in U+V$. Therefore $-x+a \in F \cap(U+V) \subseteq F \cap W$. Thus $F \cap W \neq \phi$. Therefore $z \in M^{*}-C l(F)=F$. Hence $y \in x+F$. Thus $x+F=M^{*}-C l(x+F)$. This proves that $x+F$ is $M^{*}$-closed set in $X$.

(2) Assume that $x \in M^{*}-C l(\lambda F)$. Let $W$ be any $M^{*}$-open neighborhood of $y=\frac{1}{\lambda} x$ in $X$. Since $X$ is $M^{*} I T V S$, there exist $M^{*}$-open sets $U$ in $K$ containing $\frac{1}{\lambda}$ and $V$ in $X$ containing $x$ such that $U . V \subseteq W$. By hypothesis, $(\lambda F) \cap V \neq \phi$. Therefore, there is an element $a \in(\lambda F) \cap V$. Thus $a \in \lambda F$ and $a \in V$. Hence $\frac{1}{\lambda} a \in F$ and $\frac{1}{\lambda} a \in \frac{1}{\lambda} V \subseteq U . V \subseteq W$. Therefore $F \cap W \neq \phi$. Hence $y \in M^{*}-C l(F)=F$. Thus $x \in \lambda F$ and thereby, $\lambda F=M^{*}-C l(\lambda F)$. Hence $\lambda F \in M^{*}-C(X)$.

Corollary 3.10. Let $\left(X_{(K)}, \tau\right)$ be an $M^{*}$-irresolute topological vector space and let $A \subseteq X$. Then $M^{*}-C l\left[x+M^{*}-C l(A)\right]=x+M^{*}-C l(A)$ for each $x \in X$.

Theorem 3.11. Let $\left(X_{(K)}, \tau\right)$ be an $M^{*}$-irresolute topological vector space and $S$ be a subspace of $X$. If $S$ contains a non-empty $M^{*}$-open subset of $X$, then $S$ is $M^{*}$-open in $X$.

Proof. Suppose $U$ is a non-empty $M^{*}$-open subset of $X$ such that $U \subseteq S$. By Theorem 3.3(1), for any $y \in S, U+y$ is an $M^{*}$-open subset of $X$. Since $S$ is a subspace of $X$, so also we have $U+y \subseteq S$ for any $y \in S$, Thus $S=\bigcup\{U+y: y \in S\}$ is $M^{*}$-open in $X$ being a union of $M^{*}$-open sets.

Theorem 3.12. Let $A$ be any subset of an $M^{*}$-irresolute topological vector space $X$. Then the following statements are true. 
(1) $x+M^{*}-C l(A)=M^{*}-C l(x+A)$ for any $x \in X$.

(2) $M^{*}-C l(\lambda A)=\lambda\left[M^{*}-C l(A)\right]$ for any non-zero scalar $\lambda$.

Proof. (1) By applying Theorem 3.9(1), $x+M^{*}-C l(A)$ is $M^{*}$-closed. Hence $x+A \subseteq$ $x+M^{*}-C l(A) \quad$ implies $\quad M^{*}-C l(x+A) \subseteq$ $x+M^{*}-C l(A)$. For the reverse inclusion, let $z \in x+M^{*}-C l(A)$. Then $z=x+y$, for some $y \in M^{*}-C l(A)$. Let $W$ be any $M^{*}$-open neighborhood of $z$ in $X$. Then, there exist $M^{*}$-open neighborhoods $U$ and $V$ of $x$ and $y$ respectively in $X$ such that $U+V \subseteq W$. Since $y \in M^{*}-C l(A), \quad A \cap V \neq \phi . \quad$ Consider $\quad a \in A \cap V$. Then $\quad x+a \in(x+A) \cap(U+V) \subseteq(x+A) \cap W$. Therefore we have $(x+A) \cap W \neq \phi$. Consequently, $z \in M^{*}-C l(x+A) . \quad$ Thus $\quad x+M^{*}-C l(A)$ $\subseteq M^{*}-C l(x+A)$ Hence, $x+M^{*}-C l(A)=M^{*}-C l(x+A)$

(2) By Theorem 3.9(2), $\lambda\left[M^{*}-C l(A)\right]$ is $M^{*}$-closed. Therefore $\lambda A \subseteq \lambda\left[M^{*}-C l(A)\right]$ implies that $\quad M^{*}-C l(\lambda A) \subset \lambda\left[M^{*}-C l(A)\right]$. Next, let $x \in M^{*}-C l(A)$ and let $W$ be any $M^{*}$-open neighborhood of $z=\lambda x$ in $X$. Then we get $M^{*}$-open sets $U$ in $K$ containing $\lambda$ and $V$ in $X$ containing $x$ such that $U . V \subseteq W$. Since $x \in M^{*}-C l(A)$, there is an element $a \in A \cap V$ and thus

$y=\lambda a \in(\lambda A) \cap(\lambda V) \subseteq(\lambda A) \cap(U V) \subseteq(\lambda A) \cap W$.

Hence $(\lambda A) \cap W \neq \phi$. Therefore it follows that $z=\lambda x \in M^{*}-C l(\lambda A)$.

Thus

$\lambda\left[M^{*}-C l(A)\right] \subseteq M^{*}-C l(\lambda A)$. Hence the assertion follows.

Theorem 3.13. Let $\left(X_{(K)}, \tau\right)$ be an $M^{*}$-irresolute topological vector space. Let $A$ and $B$ be subsets of $X$. Then prove that $M^{*}-C l(A)+M^{*}-C l(B) \subseteq M^{*}-C l(A+B)$.

Proof. Let $x \in M^{*}-C l(A)$ and $y \in M^{*}-C l(B)$. Let $W$ be an $M^{*}$-open neighborhood of $x+y$. Then there exist $M^{*}$-open neighborhoods $U$ and $V$ of $x$ and $y$ respectively, such that $U+V \subseteq W$. Since, $x \in M^{*}-C l(A), \quad y \in M^{*}-C l(B), \quad$ there are $a \in A \cap U \quad$ and $\quad b \in B \cap V$. Then, $a+b \in(A+B) \cap(U+V) \subseteq(A+B) \cap W$. Thus we have $(A+B) \cap W \neq \phi$. This implies that $x+y \in M^{*}-C l(A+B)$. Hence eventually we obtain $M^{*}-C l(A)+M^{*}-C l(B) \subseteq M^{*}-C l(A+B)$.

Theorem 3.14. Let $\left(X_{(K)}, \tau\right)$ be an $M^{*}$-irresolute topological vector space. For given $y \in X$ and $\lambda \in K$ with $\lambda \neq 0$, each translation mapping $T_{y}: X \rightarrow X \quad$ defined by $T_{y}(x)=x+y \quad$ and multiplication mapping $M_{\lambda}: X \rightarrow X$ defined by $M_{\lambda}(x)=\lambda x$, where $x \in X$, is $M^{*}$-homeomorphism onto itself.

Proof. First, we show that $T_{y}: X \rightarrow X$ is $M^{*}$-homeomorphism. It is obviously bijective. By Theorem 3.2 (1), $T_{y}$ is $M^{*}$-irresolute. Moreover, $T_{y}$ is pre $-M^{*}$-open because for any $M^{*}$-open $U$, by Theorem 3.3 (1), $T_{y}(U)=U+y \quad$ is $\quad M^{*}$-open. Similarly, we can prove that $M_{\lambda}$ is $M^{*}$-homeomorphism.

Theorem 3.15. Let $\left(X_{(K)}, \tau\right)$ be an $M^{*}$-irresolute topological vector space. Then any $M^{*}$-open subspace of $X$ is $M^{*}$-closed in $X$.

Proof. Let $G$ be an $M^{*}$-open subspace of $X$. Then by Theorem 3.3 (1), for any $x \in X \mathbf{B} G, G+x$ is $M^{*}$-open. We also clearly have $x \in G+x \subseteq X$ B $G$. Then, $Z=\bigcup\{G+x: x \in X$ B $G\}=X$ B $G$ being a union of $M^{*}$-open sets is $M^{*}$-open. Therefore, $G=X \mathbf{B} Z$ is $M^{*}$-closed.

Theorem 3.16. Let $\left(X_{(K)}, \tau\right)$ be an $M^{*}$-irresolute topological vector space and $B$ be an $M^{*}$-open set in $X$, then for any subset $A$ of $X$, we have $A+B=M^{*}-C l(A)+B$.

Proof. Since we know that $A \subseteq M^{*}-C l(A)$, so $A+B \subseteq s^{*} g-C l(A)+B . \quad$ Conversely, let $y \in M^{*}-C l(A)+B$ and write $y=x+b$, where 
$x \in M^{*}-C l(A)$ and $\quad b \in B$. There exists an $M^{*}$-open neighborhood $V$ of zero such that $T_{b}(V)=V+b \subseteq B . \quad$ Now, $V \quad$ is $\quad M^{*}$-open neighborhood of 0 in $X$, this gives that $-V$ is also $M^{*}$-open neighborhood of 0 in $X$. Then $x-V$ is an $M^{*}$-open neighborhood of $x$. Since $x \in M^{*}-C l(A)$, so, there exists an element $a \in A \cap(x-V)$. We know that $y=x+b=a-a+x+b \in a+V+b \subseteq A+B$.

Therefore, $M^{*}-C l(A)+B \subseteq A+B$. Hence, consequently we obtain $A+B=M^{*}-C l(A)+B$.

Theorem 3.17. Let $\left(X_{(K)}, \tau\right)$ be an $M^{*}$-irresolute topological vector space. Then the scalar multiple of $M^{*}$-closed set is $M^{*}$-closed.

Proof. Let $B$ be an $M^{*}$-closed set in $X$ and let $\lambda \in K-\{0\}$. Then $X-B$ is $M^{*}$-open set in $X$. Now $M_{\lambda}(X-B)=\lambda(X-B)=\lambda X-\lambda B=$ $X-\lambda B \in M^{*}-O(X)$. Therefore, $\lambda B \in M^{*}-C(X)$.

Definition 3.18. A topological space $(X, \tau)$ is said to be $M^{*}$-compact if every cover of $X$ by $M^{*}$-open sets of $X$ has a finite sub cover. A subset $A$ of $X$ is said to be $M^{*}$-compact relative to $X$ if every cover of $A$ by $M^{*}$-open sets of $X$ has a finite sub cover.

Theorem 3.19. Let $\left(X_{(K)}, \tau\right)$ be an $M^{*}$-irresolute topological vector space and let $A$ be any $M^{*}$-compact set in $X$. Then prove that $x+A$ is $M^{*}$-compact for each $x \in X$.

Proof. Let $\Omega=\left\{U_{\alpha}: \alpha \in \Lambda\right\}$ be an $M^{*}$-open cover of $x+A$. Then $A \subseteq \bigcup\left\{-x+U_{\alpha}: \alpha \in \Lambda\right\}$ and $\left\{-x+U_{\alpha}: \alpha \in \Lambda\right\} \subseteq M^{*}-O(X)$. By hypothesis, $A \subseteq \bigcup\left\{-x+U_{\alpha}: \alpha \in \Lambda_{0}\right\}$ for some finite $\Lambda_{0} \subseteq \Lambda$. Whence we find that $x+A \subseteq \bigcup\left\{U_{\alpha}: \alpha \in \Lambda_{0}\right\}$. This shows that $x+A$ is $M^{*}$-compact. Hence, the proof is complete.

Theorem 3.20. Let $\left(X_{(K)}, \tau\right)$ be an $M^{*}$-irresolute topological vector space. The scalar multiple of $M^{*}$-compact set is $M^{*}$-compact.

Proof. Let $A$ be an $M^{*}$-compact subset of $X$. If $\lambda=0$ we are nothing to prove. Assume $\lambda \in K-\{0\}$. Let $\Psi=\left\{U_{\alpha}: \alpha \in \Lambda\right\}$ be an $M^{*}$-open cover of $\lambda A$. Then $A \subseteq\left(\frac{1}{\lambda}\right)(\cup \Psi)=\left(\frac{1}{\lambda}\right)\left(\bigcup\left\{U_{\alpha}: \alpha \in \Lambda\right\}\right)=$

$\cup\left\{\left(\frac{1}{\lambda}\right) U_{\alpha}: \alpha \in \Lambda\right\}$.

Since $\left\{U_{\alpha}: \alpha \in \Lambda\right\} \subseteq M^{*}-O(X)$. and $\quad\left(X_{(K)}, \tau\right) \quad$ is M*ITVS, so we obtain $\left\{\left(\frac{1}{\lambda}\right) U_{\alpha}: \alpha \in \Lambda\right\} \subseteq M^{*}-O(X)$, By hypothesis $A$ is $M^{*}$-compact, therefore there exists a finite subset $\Lambda_{0} \subseteq \Lambda$ such that $A \subseteq \bigcup\left\{\left(\frac{1}{\lambda}\right) U_{\alpha}: \alpha \in \Lambda_{0}\right\}$. This implies that $\lambda A \subseteq \bigcup\left\{U_{\alpha}: \alpha \in \Lambda_{0}\right\}$. Hence $\lambda A$ is $M^{*}$-compact.

Definition 3.21. A mapping $f:\left(X_{(K)}, \tau_{X}\right) \rightarrow\left(Y_{(K)}, \tau_{Y}\right)$ is said to be linear if $f(\alpha x+\beta y)=\alpha f(x)+\beta f(y)$, for all $x, y \in X$ and $\alpha, \beta \in K$.

Definition 3.22. A mapping $f: X \rightarrow K$ is called linear functional if $f(\alpha x+\beta y)=\alpha f(x)+\beta f(y)$, for all $x, y \in X$ and $\alpha, \beta \in K$. The kernel of $f$ is defined by $\operatorname{Ker}(f)=\{x \in X: f(x)=0\}$.

Theorem 3.23. Let $f:\left(X_{(K)}, \tau_{X}\right) \rightarrow\left(Y_{(K)}, \tau_{Y}\right)$ be a linear mapping such that $f$ is $M^{*}$-irresolute at 0 . Then $f$ is $M^{*}$-irresolute on $X$.

Proof. Let $x$ be any non-zero element of $X$ and $V$ be any $M^{*}$-open set in $Y$ containing $f(x)$.

Since translation of a $M^{*}$-open set in an $M^{*}$-irresolute topological vector spaces is $M^{*}$-open, $\quad V-f(x)$ is $M^{*}$-open set in $Y$ containing 0 . Since $f$ is $M^{*}$-irresolute at 0 , there exists an $M^{*}$-open set $U$ in $X$ containing 0 such that $f(U) \subseteq V-f(x)$. Furthermore, linearity of $f$ implies that $f(x+U) \subseteq V$. By Theorem 3.3 (1), $x+U$ is $M^{*}$-open and hence $f$ is $M^{*}$-irresolute at $x$. By hypothesis, $f$ is $M^{*}$-irresolute at 0 . This reflects that $f$ is $M^{*}$-irresolute. 
Corollary 3.24. Let $\left(X_{(K)}, \tau\right)$ be an $M^{*}$-irresolute topological vector space. Let $f: X \rightarrow K$ be a linear functional which is $M^{*}$-irresolute at 0 . Then the set $F=\{x \in X: f(x)=0\}$ is $M^{*}$-closed.

\section{CHARACTERIZATIONS OF $M^{*}$-IRRESOLUTE}

\section{TOPOLOGICAL VECTOR SPACES}

In this section we give some characterizations of $M^{*}$-irresolute topological vector space.

Theorem 4.1. Let $(X, \tau)$ be an $M^{*}$-irresolute topological vector space. For $x \in X$, the following assertions are true:

(1) If $U \in M^{*}-N(x)$, then $x \in U$.

(2) If $U \in M^{*}-N(x)$ and $V$ is a neighborhood of $x$, then $U \cap V \in M^{*}-N(x)$.

(3) If $U \in M^{*}-N(x)$, then there exists $V \in M^{*}-N(x)$ such that $U \in M^{*}-N(y)$, for all $y \in V$.

(4) If $U \in M^{*}-N(x)$ and $U \subseteq V$, then $V \in M^{*}-N(x)$.

(5) If $U \in M^{*}-N(0)$, then $\lambda U \in M^{*}-N(0)$ for every non-zero element $\lambda \in R$.

(6) If $U \in M^{*}-N(x)$ and $V$ is an $M^{*}$-neighborhood of $x$, then $U \cap V \in M^{*}-N(x)$.

(7) $U \in M^{*}-N(0)$ if and only if $x+U \in M^{*}-N(x)$.

Proof. We will prove (2), (5) and (7) while the proofs of others follow easily.

(2) If $U$ is an $M^{*}$-neighborhood of $x$, and $V$ is a neighborhood of $x$, then there is an $M^{*}$-open subset $A$ and an open set $B$ such that $x \in A \subseteq U$ and $x \in B \subseteq V . \quad$ Then $x \in A \cap B \subseteq U \cap V \quad$ and $\tau \subseteq M^{*}-O(X)$. Thus $A \cap B \in M^{*}-O(X)$. Therefore $U \cap V$ is an $M^{*}$-neighborhood of $x$.

(5) Let $U$ be an $M^{*}$-neighborhood for zero. Then there exists an $M^{*}$-open neighborhood $V$ of zero such that $V \subseteq U$. Since the map $M_{\lambda}: X \rightarrow X$, defined by $M_{\lambda}(x)=\lambda x$, is $M^{*}$-irresolute. The inverse map $M_{\lambda} ; X \rightarrow X$, defined by $M_{\lambda}(x)=\frac{1}{\lambda} x$, is also an $M^{*}$-irresolute. Thus $M_{\lambda}$ is $M^{*}$-homeomorphism, for each $\lambda \in R-\{0\}$. Hence $M_{\lambda}(V)=\lambda V$ is an $M^{*}$-open neighborhood of zero. Furthermore, clearly $\lambda V \subseteq \lambda U$. Thus consequently $\lambda U \in M^{*}-N(0)$.

(7) Suppose $U$ is an $M^{*}$-neighborhood for zero. Then there exists an $M^{*}$-open neighborhood $V$ of zero such that $V \subseteq U$. Since the map $T_{x}: X \rightarrow X$, defined by $T_{x}(y)=y+x$, is $M^{*}$-irresolute. The inverse map $T_{x} ; X \rightarrow X$, defined by $T_{x}(y)=x-y$, is also an $M^{*}$-irresolute. Thus $T_{x}$ is $M^{*}$-homeomorphism, for each $x \in X$. Hence $T_{x}(V)=x+V$ is an $M^{*}$-open neighborhood for a point $x$. Clearly $x+V \subseteq x+U$. Thus $x+U \in N_{x}$. The converse can be proved similarly.

Definition 4.2. A subset $A$ of a topological vector space $X$ is called balanced if and only if $\lambda A \subseteq A$ for each $\lambda \in R$ such that $|\lambda| \leq 1$.

Definition 4.3. A subset $A$ of a topological vector space $X$ is called absorbing if for all $x \in X$ there exists a number $\varepsilon>0$ such that $\lambda x \in A$ for $|\lambda| \leq \varepsilon$.

Definition 4.4. A set $C$ of a topological vector space $X$ is said to be convex, if and only if it contains all segments between its points: $x \in C$, $y \in C$, for $t \in[0,1]$ implies $t x+(1-t) y \in C$, or equivalently $t C+(1-t) C \subseteq C$, for all $t \in[0,1]$. A set $C$ of a topological vector space $X$ is said to be absolutely convex if it is both convex and balanced.

Theorem 4.5. Let $\left(X_{(K)}, \tau\right)$ be an $M^{*}$-irresolute topological vector space. If a subset $C$ of $X$ is convex, then $\mathrm{M}^{*}-\mathrm{Cl}(C)$ is also convex.

Proof. The convexity of $C$ implies $t C+(1-t) C \subseteq C$. By Theorem 3.12 (2), and Theorem 3.13, it follows immediately that $t\left[M^{*}-C l(C)\right]+(1-t)\left[M^{*}-C l(C)\right]=M^{*}-C l(t C)+$ $M^{*}-C l[(1-t) C] \subseteq M^{*}-C l[t C+(1-t) C]$ $=M^{*}-C l(C)$. Thus $t\left[M^{*}-C l(C)\right]+(1-t)\left[M^{*}-C l(C)\right] \subseteq M^{*}-C l(C)$. 
Hence we conclude that $M^{*}-C l(C)$ is convex.

Theorem 4.6. Let $\left(X_{(K)}, \tau\right)$ be an $M^{*}$-irresolute topological vector space. If a subset $C$ of $X$ is convex, then $M^{*}-\operatorname{Int}(C)$ is also convex.

Proof. By Theorem 3.7 (2), and Theorem 3.8, $t\left[M^{*}-\operatorname{Int}(C)\right]+(1-t)\left[M^{*}-\operatorname{Int}(C)\right]=$ $M^{*}-\operatorname{Int}(t C)+\quad M^{*}-\operatorname{Int}[(1-t) C] \subseteq$ $M^{*}-\operatorname{Int}[t C+(1-t) C]=M^{*}-\operatorname{Int}(C) . \quad$ Therefore $M^{*}-\operatorname{Int}(C)$ is convex.

Theorem 4.7. Let $\left(X_{(R)}, \tau\right)$ be an $M^{*}$-irresolute topological vector space. Then the following statements are equivalent:

(a) Every $M^{*}$-neighborhood $U$ of zero is absorbing.

(b) For every $M^{*}$-neighborhood $U$ for zero, there exists a balanced set $V \in M^{*}-N(0)$ such that $V \subseteq U$

Proof. (a) Suppose $U$ is an $M^{*}$-neighborhood for zero. Then there exists an $M^{*}$-open subset $V \in M^{*}-N(0)$ such that $V \subseteq U$. By hypothesis $X$ is an $M^{*} I T V S$. So there exist $M^{*}$-open sets $V_{1}$ of $R$ containing zero and $V_{2}$ of $X$ containing zero such that $V_{1} \cdot V_{2} \subseteq V$. The set $V_{1}$ contains an open interval of the form $(-\varepsilon, \varepsilon)$ for some $\varepsilon>0$. Therefore $t x \in V \subseteq U$ for all $t \in(-\varepsilon, \varepsilon)$ and for all $x \in V_{2}$. This implies $U$ is absorbing.

(b) Let $U$ be an $M^{*}$-neighborhood of zero. By hypothesis $X$ is an $M^{*} I T V S$. So there exist $M^{*}$-open sets $V_{1}$ of $R$ containing zero and $V_{2}$ of $X$ containing zero such that $V_{1} \cdot V_{2} \subseteq U$. Then there exists $\varepsilon>0$ such that $(-\varepsilon, \varepsilon) \subseteq V_{1}$. Define $W=\bigcup\left\{t V_{2}: t \in R,|t|<\varepsilon\right\}$. Since $t V_{2} \quad$ is an $M^{*}$-neighborhood of zero, for $t \neq 0$ and $t V_{2} \subseteq U$ for $t \in(-\varepsilon, \varepsilon)$. Thus $W$ is an $M^{*}$-neighborhood for zero and $W \subseteq U$. Now we have to show that $W$ is balanced. Let $r \in R$ such that $|r| \leq 1$. Let $t \in(-\varepsilon, \varepsilon)$ and $x \in V_{2}$. Since $|r t|=|r||t| \leq t<\varepsilon$. Thus $r(t x)=(r t) x \in(-\varepsilon, \varepsilon) . V_{2} \subseteq W$. This shows that $r W \subseteq W$. Therefore $W$ is balanced.
Theorem 4.8. Let $X$ be an $M^{*}$-irresolute topological vector space. Then $M^{*}-C l(A)=\bigcap\left\{A+U: U \in M^{*}-N(0)\right\}$.

Proof. Assume $x \in M^{*}-C l(A)$, and let $U$ be an $M^{*}$-neighborhood of zero. Then by Theorem 4.7(b), there exits a balanced neighborhood $V$ for zero such that $V \subseteq U$. Thus $x+V$ is an $M^{*}$-neoghborhood for $x$ and $x \in M^{*}-C l(A)$, so $(x+V) \cap A \neq \phi$. Take $a \in(x+V) \cap A$. Then $a \in x+V$ and $a \in A$. Let $a=x+v$ for some $v \in V$. Since $V$ is balanced, so $A-V=A+V$. Take $x=a+(-v) \in A-V$ implies $x \in A+V \subseteq A+U$. Thus $x \in A+U$, for any $M^{*}$-neighborhood $U$ of zero. Therefore, we obtain $M^{*}-C l(A) \subseteq \bigcap\left\{A+U: U \in M^{*}-N(0)\right\}$.

Conversely if $x \notin M^{*}-C l(A)$, then there exists a balanced neighborhood $U$ for zero such that $(x+U) \cap A=\phi$. Thus $x \notin A-U=A+U$. It follows that $\bigcap\left\{A+U: U \in M^{*}-N(0)\right\} \subseteq M^{*}-C l(A)$. Thus we get $M^{*}-C l(A)=\bigcap\left\{A+U: U \in M^{*}-N(0)\right\}$.

Theorem 4.9. Let $X$ be an $M^{*} I T V S$. Then the following assertions are true:

(a) For every $U \in M^{*}-N(0)$, there exists symmetric set $V \in M^{*}-N(0)$ such that $V+V \subseteq U$.

(b) For every $U \in M^{*}-N(0)$, there exits an $M^{*}$-closed balanced set $V \in M^{*}-N(0)$ such that $V \subseteq U$.

(c) For every $U \in M^{*}-N(0)$, there exists symmetric set $V \in M^{*}-N(0)$ such that $V+V+V \subseteq U$.

Proof. (a) Assume $U \in M^{*}-N(0)$. By hypothesis $X$ is an $M^{*} I T V S$. There exist $M^{*}$-open neighborhoods $V_{1}$ and $V_{2}$ for zero in $X$ such that $V_{1}+V_{2} \subseteq U$. Let $V=V_{1} \cap\left(-V_{1}\right) \cap V_{2} \cap\left(-V_{2}\right)$. Then $V$ is symmetric $M^{*}$-open neighborhood of zero and $V+V \subseteq V_{1}+V_{2} \subseteq U$.

(b) Let $U$ be an $M^{*}$-neihborhood of zero in $X$. By part (a) there is $M^{*}$-neihborhood $V$ for zero with $V+V \subseteq U$. By Theorem 4.7 (b), there exits $M^{*}$-neihborhood $W$ for zero which is balanced and $W \subseteq V . \quad$ By $\quad$ Theorem 4.8, 
$M^{*}-C l(W) \subseteq W+V \subseteq V+V \subseteq U$. This shows that

$U$ contains $M^{*}$-closed neighborhood of zero.

(c) Follows easily from (a).

Definition 4.10. A topological space $(X, \tau)$ is called $M^{*}-$ Hausdorff, if each two distinct points $x$ and $y$ in $X$, there exist disjoint $M^{*}$-open sets $U$, $V$ such that $x \in U$ and $y \in V$.

Now we give some properties of $M^{*}$-Hausdorff space.

Theorem 4.11. Let $X$ be an $M^{*} I T V S$. Then the following statements are equivalent.

(a) $X$ is $M^{*}$-Hausdorff.

(b) If $x \in X, x \neq 0$, then there exists $U \in M^{*}-N(0)$ such that $x \notin U$.

(c) If $x, y \in X, x \neq y$, there exists $V \in M^{*}-N(x)$ such that $y \notin V$.

Proof. By continuity of translation, it is sufficient to prove the equivalence between (a) and (b) only.

$(\mathrm{a}) \Rightarrow(\mathrm{b})$ : Assume $x$ be a non-zero vector belongs to $X$. Therefore there are disjoint $M^{*}$-open sets $U, V \subseteq X$ such that $0 \in U$ and $x \in V$. Thus $U \in M^{*}-N(0), V \in M^{*}-N(x)$ and $x \notin U$.

(b) $\Rightarrow(\mathrm{a})$ : Let $x, y \in X$ be such that $x-y \neq 0$. Then there exists $U \in M^{*}-N(0)$ such that $x-y \notin U$. By Theorem 4.9 (a), there exists $M^{*}$-neihborhood $W$ of zero such that $W+W \subseteq U$. By Theorem 4.7 (b), $W$ can be assumed to be balanced. Let $V_{1}=x+W$ and $V_{2}=y+W$. We note that $V_{1} \in N_{x}$, $V_{2} \in N_{y}$ and $V_{1} \cap V_{2}=\phi$, since if $z \in V_{1} \cap V_{2}$ then $z-x \in W$ and $z-y \in W$. Since $W$ is balanced, so $-(z-x) \in W$. It follows that $x-y=(z-y)+\lfloor-(z-x)\rfloor \in W+W \subseteq U$, which is a contradiction. So we must have $V_{1} \cap V_{2}=\phi$. Finally, the space $X$ is $M^{*}$-Hausdorff.

The following result follows from Theorem 4.11.

Corollary 4.12. Let $X$ be an $M^{*} I T V S$. Then the following statements are equivalent.
(a) $X$ is $M^{*}$-Hausdorff.
(b) $\cap\left\{U: U \in M^{*}-N(0)\right\}=\{0\}$.
(c) $\cap\left\{U: U \in M^{*}-N(x)\right\}=\{x\}$.

Theorem 4.12. An $M^{*} I T V S X$ is $M^{*}$-Hausdorff if and only if one-point set in $X$ is $M^{*}$-closed in $X$.

Proof. Let $x \in X$ and $y \in X \mathbf{B}\{x\}$. Then $y-x \neq 0$, and by assumption, there exists $U \in M^{*}-N(0)$ such that $y-x \notin U$. By Theorem 4.9 (b), there exists an $M^{*}$-closed and balanced set $V \in N_{0}$ such that $V \subseteq U$. It follows that $y-x \notin V$ that is $y-x \in X \mathbf{B} V$. Thus $y \in(X \mathbf{B} V)+\{x\}$. But $(X \mathbf{B} V)+\{x\}$ is $M^{*}$-open, since $V$ is $M^{*}$-closed, and $(X \mathbf{B} V)+\{x\} \subseteq X \mathbf{B}\{x\}$. This shows that $X \mathbf{B}\{x\}$ is $M^{*}$-open. For the converse, let $x \in X$ and assume that $\{x\}$ is $M^{*}$-closed. Then by Theorem 4.8, $\{x\}=M^{*}-C l(\{x\})=$

$\bigcap\left\{U+\{x\}: U \in M^{*}-N(0)\right\}$

$=\bigcap\left\{V: V \in M^{*}-N(x)\right\}$, where $V=U+\{x\} \in M^{*}-N(x)$. Then by Corollary 4.12, $X$ is $M^{*}$-Hausdorff. We know that translation is an $M^{*}$-homeomorphism and as a consequence of Theorem 4.12, we have the following result.

Corollary 4.13. An $M^{*} I T V S X$ is $M^{*}$-Hausdorff if and only if $\{0\}$ is $M^{*}$-closed in $X$.

Theorem 4.14. Let $C, K$ be disjoint sets in an $M^{*} I T V S X$ with $C M^{*}$-closed, $K M^{*}$-compact. Then there exists $U \in M^{*}-N(0)$ with $(K+U) \cap(C+U)=\phi$.

Proof. If $K=\phi$, there is nothing to prove. Otherwise, let $x \in K$ by the invariance with translation; we can assume $x=0$. Then $X \mathbf{B} C$ is an $M^{*}$-open set of zero. Since addition is $M^{*}$-irresolute and $M^{*}$-continuous, by $0+0+0=0$, there is an $M^{*}$-open neighborhood $U \in M^{*}-N(0)$ such that $3 U=U+U+U \subseteq X$ B $C$. By defining $U=U \cap(-U) \subseteq U$ we have that $U$ $M^{*}$-open symmetric and $3 U=U+U+U \subseteq X$ B $C$. This means that $\phi=\{3 x: x \in U\} \cap C=$ $\{2 x: x \in U\} \cap\{y-x: y \in C, x \in U\} \supseteq \supseteq U \cap(C+U)$. This concludes the proof for a single point. Sine $K$ is 
$M^{*}$-compact, then repeating the above argument for all $x \in K$, we obtain symmetric $M^{*}$-open sets $V_{x}$ such that $\left(x+2 V_{x}\right) \cap\left(C+V_{x}\right)=\phi$. The sets $\left\{V_{x}: x \in K\right\}$ are an $M^{*}$-open covering of $K$, but $K$ is $M^{*}$-compact. Hence there is a finite number of points $x_{i} \in K, \quad i=1,2, \ldots, n, \quad$ such that $K \subseteq \bigcup_{i=1}^{n}\left(x_{i}+V_{x_{i}}\right) . \quad$ Define the $M^{*}$ open neighborhood $V$ of zero by $V=\bigcap_{i=1}^{n} V_{x_{i}}$. Then we get $(K+V) \cap(C+V) \subseteq \quad \bigcup_{i=1}^{n}\left(x_{i}+V_{x_{i}}+V\right) \cap(C+V) \subseteq$ $\bigcup_{i=1}^{n}\left(x_{i}+2 V_{x_{i}}\right)\left(C+V_{x_{i}}\right)=\phi$. This completes the proof.

Lemma 4.15. If $U$ is a $M^{*}$-open set and $U \cap A=\phi$, then $U \cap M^{*}-C l(A)=\phi$.

Proof. Suppose that there exists an $x \in U \cap M^{*}-C l(A)$. Then $x \in M^{*}-C l(A)$ and $U$ is an $M^{*}$-open neighborhood of $x$ and $X \mathbf{B} U$ is $M^{*}$-closed set containing $A$, hence $M^{*}-C l(A) \subseteq X$ B $U$ and $x \notin M^{*}-C l(A)$ which is contradiction, hence $U \cap M^{*}-C l(A)=\phi$.

Corollary 4.16. Let $C, K$ be disjoint sets in an $M^{*}$ ITVS $\quad X \quad$ with $C M^{*}$-closed, $K$ $M^{*}$-compact. Then there exists $U \in M^{*}-N(0)$ with $\left[M^{*}-C l(K+U)\right] \cap(C+U)=\phi$.

Proof. By Theorem 4.14, there exists $U \in M^{*}-N(0)$ such that $(K+U) \cap(C+U)=\phi$. $C+U=\bigcup\{y+U: y \in C\}$ is $M^{*}$-open set being a union of $M^{*}$-open sets. Now by Lemma 4.15, we obtain $\left[M^{*}-C l(K+U)\right] \cap(C+U)=\phi$.

Theorem 4.17. Let $X$ be an $M^{*} I T V S$. Let $f: X \rightarrow K$ be a non-zero linear map. Then $f(G)$ is $M^{*}$-open in $K$ whenever $G$ is $M^{*}$-open in $X$.

Proof. Let $G$ be a nonempty $M^{*}$-open set. Then once can assume that there is $x_{0} \in X-\{0\}$ such that $f\left(x_{0}\right)=1$. For any $a \in G$, it is required to show that $f(a) \in M^{*}-\operatorname{Int}[f(G)]$. Since $G \in M^{*}-N(a)$ by Theorem 4.1 we have $G-a \in M^{*}-N(0)$. By
Theorem 4.7 (a) $G-a$ is absorbing, that is, absorbs $x_{0}$, namely there exists an $\varepsilon>0$ such that $\lambda x_{0} \in G-a$ whenever $\lambda \in R$ with $|\lambda| \leq \varepsilon$. Now for any $\beta \in R \quad$ with $\quad|\beta-f(a)|<\varepsilon \quad$ we have $(\beta-f(a)) x_{0} \in G-a$, hence $f\left\lfloor(\beta-f(a)) x_{0}\right\rfloor \in f(G-a)$. Since $f$ is linear. This implies that $(\beta-f(a)) f\left(x_{0}\right) \in f(G-a)$. So we get $\quad(\beta-f(a))(1) \in f(G-a)=f(G)-f(a)$. This implies that $\beta \in f(G)$. Thus shows that $(f(a)-\varepsilon, f(a)+\varepsilon) \subseteq f(G)$. Since $(f(a)-\varepsilon, f(a)+\varepsilon)$ is $\theta$-open in $K$. Thus $f(a) \in \operatorname{Int}_{\theta}[f(G)] \subseteq M^{*}-\operatorname{Int}[f(G)] ; \quad$ hence consequently $f(G)=M^{*}-\operatorname{Int}[f(G)]$.

Lemma 4.18. [Horvath;1966]. Let $X$ be vector space and $\phi \neq K \subseteq X$. For $a \in K$, the following statements are equivalent:

(1) $a$ is an extreme point of $K$.

(2) If $x, y \in K$ are such that $a=\frac{1}{2}(x+y)$, then $a=x=y$.

(3) Let $x, y \in K$ be such that $x \neq y$, let $\lambda \in(0,1)$ and $a=\lambda x+(1-\lambda) y$. Then we have either $\lambda=0$ or $\lambda=1$.

Theorem 4.19. Let $X$ be an $M^{*} I T V S$ and $C$ be a convex subset of $X$. Then $\left[M^{*}-\operatorname{Int}(C)\right] \cap(\partial C)=\phi$. Proof. If $M^{*}-\operatorname{Int}(C)=\phi$, then the result is trivial. Suppose that $M^{*}-\operatorname{Int}(C) \neq \phi \quad$ and let $x \in M^{*}-\operatorname{Int}(C)$. Then there exists $V \in M^{*}-N(0)$ such that $x+V \subseteq C$. As the map $\Phi: \mathfrak{R} \rightarrow X$, where $\Phi(\mu)=\mu x$ is continuous at $\mu=1$, for this $M^{*}$-Ineighborhood $x+V$ there is an $r>0$ such that $\mu x \in x+V$ whenever $|\mu-1| \leq r$. In particular, we have $(1+r) x \in x+V \subseteq C$ and $(1-r) x \in x+V \subseteq C$. Now consider $x=\lambda(1+r) x+(1-\lambda)(1-r) x$ and set $\lambda=\frac{1}{2}$. Consequently, we have $x=\frac{1}{2}(1+r) x+\left(1-\frac{1}{2}\right)(1-r) x$, which implies that $x$ is not an extreme point of $K$. 
Theorem 4.20. Let $X$ be an $M^{*} I T V S$ and $W$ an $M^{*}$-neighborhood of 0 . Then there is an $M^{*}$-neighborhood $U$ of 0 such that $M^{*}-C l(U) \subseteq W$. Equivalently, if $C$ is a $M^{*}$-closed subset of $X$ and $x$ a point of $X$ outside $C$ then there are disjoint $M^{*}$-open sets $U_{1}$ and $U_{2}$ with $x \in U_{1}$ and $C \subseteq U_{2}$.

Proof. Let $x$ be a point outside an $M^{*}$-closed set $C \subseteq X$. We will produce an $M^{*}$-open set $U$ containing $x$ with $M^{*}-C l(U) \cap C=\phi$; then $U_{1}=U$ and $U_{2}=X \mathbf{B} M^{*}-C l(U)$, the complement of the $M^{*}$-closure of $U$, are disjoint $M^{*}$-open sets with $x \in U_{1}$ and $C \subseteq U_{2}$, as desired. We know that $X$ looks the same everywhere, so we may work with $x=0$. Let $W$ be the complement of $C$. Then $W$ is an $M^{*}$-open set with $0 \in W$. By hypothesis $X$ is $M^{*} I T V S$. So by Theorem 4.9 (a), there exists an $M^{*}$-open subset $U$ of 0 such that $U+U \subseteq W$. This means that $U+U$ is disjoint from $C$. Equivalently, $U$ is disjoint from $C \mathbf{B} U$. For otherwise there would be an $x \in U$ which could be expressed as $c-y$ with $c \in C$ and $y \in U$, which would imply that $c=x+y \in U+U \subseteq W$ is in $W$. Now the set $-U$ is $M^{*}$-open because the map $X \rightarrow X: \quad x \rightarrow(-1) x=-x \quad$ is $\quad$ an $M^{*}$-homeomorphism, and hence so are all its translates $x-U . \quad$ So the set $U_{2}=C \mathbf{B} U=\bigcup\{c-U: c \in C\} \quad$ is $\quad M^{*}$-open, being the union of $M^{*}$-open sets. Thus we have found an $M^{*}$-open set $U_{1}=U$ containing 0 and an $M^{*}$-open set $U_{2}$, disjoint from $U_{1}$, with $C \subseteq U_{2}$. Theorem 4.21. Let $\left(X_{(K)}, \tau\right)$ be an $M^{*} I T V S$. Then every $M^{*}$-open subspace $S$ of $X$ is also an $M^{*} I T V S$.

Proof. Let $W$ be an $M^{*}$-open neighborhood of $x+y$ in $S$ where $x, y$ are two distinct points in $S$. Since $S$ is an $M^{*}$-open subspace of $X$, then $W$ is an $M^{*}$-open neighborhood of $x+y$ in $X$, and by definition of $M^{*} I T V S$, there exist $M^{*}$-open neighborhoods $U$ of $x$ in $X$ and $V$ of $y$ in $X$ such that $U+V \subseteq W$. Then the sets $G=U \cap S$ and
$H=V \cap S$ are $M^{*}$-open neighborhoods of $x$ and $y$ in $S$ such that $G+H \subseteq U+V \subseteq W$. Now suppose $\lambda \in K, x \in S$ and let $W$ be an $M^{*}$-open neighborhood of $\lambda x$ in $S$. Since $S$ is an $M^{*}$-open subspace of $X$, then $W$ is an $M^{*}$-open neighborhood of $\lambda x$ in $X$. Then there exist $M^{*}$-open neighborhoods $U$ of $\lambda$ in $K$ and $V$ of $y$ in $X$ such that $U . V \subseteq W$. Then the set $G=U \cap S$ is an $M^{*}$-open neighborhood of $\lambda$ in $K$ and the set $H=V \cap S$ is an $M^{*}$-open neighborhood of $x$ in $S$. Also $G . H \subseteq U . V \subseteq W$. Hence $S$ is an $M^{*} I T V S$.

Theorem 4.22. Let $\left(X_{(K)}, \tau\right)$ be an $M^{*} I T V S$. Then every $M^{*}$-open subspace $S$ of $X$ is $M^{*}$-closed in $X$.

Proof. By Theorem $3.3(1), S+x$ is $M^{*}$-open in $X$. Then $Y=\bigcup\{S+x: x \in X \mathbf{B} S\}$ being union of $M^{*}$-open sets is also $M^{*}$-open. Thus $S=X \mathbf{B} Y$ is $M^{*}$-closed.

Theorem 4.23. Suppose that $\left(X_{(K)}, \tau\right)$ is an $M^{*} I T V S$. If $S \subseteq X$ is a linear subspace, then so is $M^{*}-\mathrm{Cl}(S)$.

Proof. Let $S$ be a linear subspace of $X$. Thus $S+S \subseteq S$ and for all $\lambda \in K, \lambda . S \subseteq S$. By Theorem 3.13 ,

$M^{*}-C l(S)+M^{*}-C l(S) \subseteq M^{*}-C l(S+S) \subseteq M^{*}-C l(S)$. By Theorem 3.12, for every $\lambda \in K$, $\lambda\left[M^{*}-C l(S)\right]=M^{*}-C l(\lambda S) \subseteq M^{*}-C l(S)$.

Therefore, $M^{*}-C l(S)$ is linear subspace of $X$.

Definition 4.24. Suppose that $\left(X_{(K)}, \tau\right)$ is an $M^{*} I T V S$. A subset $E \subseteq X$ is said to be bounded if for all $M^{*}$-open set $V$ containing 0 , there exists $s \in R$ such that for all $t>s, E \subseteq t V$.

Theorem 4.25. Suppose that $\left(X_{(K)}, \tau\right)$ is an $M^{*} I T V S$. If $E$ is a bounded subset of $X$, then $M^{*}-C l(E)$ is bounded.

Proof. Let $W$ be an $M^{*}$-open set containing 0 , then by Theorem 4.20, there exists $U \in M^{*}-N(0)$ such that $M^{*}-C l(U) \subseteq W$. Since $E$ is bounded, so $E \subseteq t U \subseteq t\left[M^{*}-C l(U)\right] \subseteq t W$, for sufficiently large 
t. It follows that for large enough $t$, $M^{*}-C l(E) \subseteq M^{*}-C l(t U) \subseteq t\left[M^{*}-C l(U)\right] \subseteq t W$.

Thus, $M^{*}-C l(E)$ is bounded.

Theorem 4.26. Let $(X, \tau)$ be an $M^{*} I T V S$. Let $V$ be an $M^{*}$-open neighborhood of zero in $X$. Then for every sequence $\left\{r_{n}: n \in N\right\}$ of positive real numbers such that $r_{n} \rightarrow \infty, \bigcup_{n=1}^{\infty} r_{n} V=X$.

Proof. Let $x \in X$ and consider the sequence $\left\{\frac{x}{r^{n}}: n \in N\right\}$. This sequence converges to 0 by the $M^{*}$-irresoluteness of the scalar multiplication $F \times X \rightarrow X$. Thus, for sufficiently large $n, \frac{x}{r^{n}} \in V$ i.e., $x \in r_{n} V$.

Theorem 4.27. Let $(X, \tau)$ be an $M^{*} I T V S$. Then every $M^{*}$-compact set is bounded.

Proof. Let $C$ be an $M^{*}$-compact subset of $X$. We need to prove that it is bounded, namely, that for every $M^{*}$-open neighborhood $V$ of $0, K \subseteq t V$ for sufficiently large $t$. Let $V$ be an $M^{*}$-open neighborhood of 0 , then by Theorem 4.7(b), there exists a balanced $M^{*}$-open neighborhood $W$ of 0 such that $W \subseteq V$. By Theorem 4.26, $C \subseteq \bigcup_{j=1}^{\infty} n W$. Since, $C$ is $M^{*}$-compact, therefore there exists a positive integer $\mathrm{m}$ such that $C \subseteq \bigcup_{j=1}^{m} n_{j} W=n_{m} \bigcup_{j=1}^{m}\left(n_{j} / n_{m}\right) W \subseteq n_{m} W . \quad$ Thus, for all $t>n, C \subseteq n_{m} W=\left\lfloor t\left(n_{m} / t\right)\right\rfloor W \subseteq t W \subseteq t V$, which implies that $C$ is bounded.

Theorem 4.28. Let $(X, \tau)$ be an $M^{*} I T V S$. Then every Cauchy sequence in $X$ is bounded.

Proof. Let $\left\{x_{n}: n \in N\right\}$ be a Cauchy sequence in $X$. Let $W$ be an $M^{*}$-open neighborhood of zero, then by Theorem 4.9 (a), there exists an $M^{*}$-open neighborhood $V$ of 0 such that $V+V \subseteq W$. By definition of a Cauchy sequence, there exists $N$ such that for all $m, n \geq N, \quad x_{n}-x_{m} \in V$ and in particular for all $n>N, x_{n} \in x_{N}+V$. Set $s>1$ such that $x_{n} \in s V$, then for all $n>N, \quad x_{n} \in s V+V \subseteq$ $s V+s V \subseteq s W$. Since for balanced sets $s W \subseteq t W$ for $s<t$, and since every $M^{*}$-open neighborhood of zero contains a balanced neighborhood, this proves that the sequence is indeed bounded.

Definition 4.29. Let $(X, \tau)$ be a vector space over $\square$. A non-negative real-valued function $p$ defined on $X$ is a pseudonorm if it satisfies the following two conditions:

(i) $p(\lambda x)=|\lambda| p(x)$, for all $\lambda \in \square$;

(ii) $p(x+y) \leq p(x)+p(y)$, for all $x, y \in X$.

Now, we introduce the notion of locally convex $M^{*} I T V S$. Moreover, we give a necessary and sufficient condition, in terms of convex $M^{*}$-neighborhoods of 0 , for an $M^{*} I T V S$ to be locally convex.

Definition 4.30. An $M^{*} \operatorname{ITVS}(X, \tau)$ is locally convex if for all $x \in X$, every $S \in M^{*}-N(x)$ contains a convex set $U \in M^{*}-N(x)$.

Theorem 4.31. An $M^{*} I T V S \quad(X, \tau)$ is locally convex if and only if every $S \in M^{*}-N(0)$ contains a convex set $U \in M^{*}-N(0)$.

Proof. The sufficiency part is trivial. Let $S \in M^{*}-N(x)$. Then by Theorem $4.1 \quad$ (7), $S-x \in M^{*}-N(0)$ and by assumption, there exists a convex set $U \in M^{*}-N(0)$ such that $U \subseteq S-x$. Hence by Theorem 4.1 (7) again, $U+x \in M^{*}-N(x)$. As $U+x \subseteq S$ and as $U+x$ is convex, $(X, \tau)$ is a locally convex $M^{*} I T V S$.

Corollary 4.32. In a locally convex $M^{*} I T V S$ $(X, \tau)$, a pseudonorm $p$ is $M^{*}$-irresolute if and only if $p$ is $M^{*}$-irresolute at zero.

Proof. If $p$ is $M^{*}$-irresolute, then $p$ is $M^{*}$-irresolute at zero. Conversely, suppose $p$ is $M^{*}$-irresolute at 0 , and let $x \in X$ and $V \in N_{p(x)}(\square)$. Then by Theorem 4.1 (7), $V-p(x) \in N_{0}(\square)=N_{p(0)}(\square) \quad$ and thus $(-\varepsilon, \varepsilon) \subseteq V-p(x)$ for some $\varepsilon>0$. Clearly $(-\varepsilon, \varepsilon)$ being an open set in $\square$ is $M^{*}$-open set in $\square$. By assumption, there exists $U \in M^{*}-N(0)$ such that $p(U) \subseteq(-\varepsilon, \varepsilon)$ and as $p(y) \geq 0$ for all $y \in U$, $p(U) \subseteq[0, \varepsilon)$. Then by Theorem $4.1 \quad$ (7), $U+x \in M^{*}-N(x)$. For all $y \in U, \quad 0 \leq p(x+y) \leq$ 
$p(x)+p(y) \leq p(x)+\varepsilon, p(x+y) \in[0, p(x)+\varepsilon)$.

Therefore it follows that $p(U+x) \subseteq V$.

Definition 4.33. Let $A$ be an absolutely convex subset of a vector space $X$. Then the functional defined by $p(x)=\inf \{\lambda: \lambda>0, x \in \lambda A\}$ is called the gauge of $A$.

Lemma 4.34. [Horvath;1966]. In a vector space $X$, the gauge of an absolutely convex and absorbent subset is a pseudonorm.

Now, we prove a main result in which we characterize absolutely convex and absorbent $M^{*}$-neoghborhoods of zero in terms of their $M^{*}$-irresolute gauges.

Theorem 4.35. Let $p$ be a gauge of an absolutely convex and absorbent subset $U$ of an $M^{*} I T V S$ $(X, \tau)$. Then $p$ is $M^{*}$-irresolute if and only if $U$ is an $M^{*}$-neighborhood of zero.

Proof. If $p$ is $M^{*}$-irresolute, then as $(-1,1)$ is an $M^{*}$-open set in $\square . V=\{x: p(x)<1\}=p^{-1}[(-1,1)]$ is an $M^{*}$-open subset of $X$. Thus as $V \subseteq U$, $U \in M^{*}-N(0)$. Conversely, if $U \in M^{*}-N(0)$ and $\varepsilon>0$, then by Theorem 4.1 (5), $V=\varepsilon U \in M^{*}-N(0)$ and $p(x)<\varepsilon$ for all $x \in V$. Thus $p(V) \subseteq(-\varepsilon, \varepsilon)$. Hence, $p$ is $M^{*}$-irresolute at zero. By Lemma 4.34, $p$ is a pseudonorm and by Corollary 4.33, $p$ is $M^{*}$-irresolute at each $x \in X$. Therefore $p$ is $M^{*}$-irresolute.

\section{CONCLUSION}

Topological vector spaces are a fundamental notion and play an important role in various advanced branches of mathematics like fixed point theory, operator theory, etc. This paper expounds the $\mathrm{M}^{*}$-irresolute topological vector spaces. The author makes familiarity with the origin of $\mathrm{M}^{*}$-irresolute topological vector spaces and presents several basic features of $\mathrm{M}^{*}$-irresolute topological vector spaces. The author also presents the result that in an $\mathrm{M}^{*}$-irresolute topological vector space any $\mathrm{M}^{*}$-neighborhood for 0 contains an $\mathrm{M}^{*}$-neighborhood for 0 which is balanced. $\mathrm{M}^{*}$-irresolute topological vector space satisfies the separation axiom ( $\mathrm{M}^{*}$-Hausdorff $)$ and in an $\mathrm{M}^{*}$-irresolute topological vector space the $\mathrm{M}^{*}$-interior of a convex set does not intersect with the boundary of the set. The author also presents a result to characterize absolutely convex and absorbent $M^{*}$-neoghborhoods of zero in terms of their $M^{*}$-irresolute gauges.

\section{ACKNOWLEDGEMENT}

The author is highly and greatly indebted to Prince Mohammad Bin Fahd University Saudi Arabia, for providing all necessary research facilities during the preparation of this research paper.

\section{Conflict of Interest}

The author declares that this publication is not subject to conflict of interests.

\section{REFERENCES}

[1]. M.E. Abd-El Monsef, S.N. El-Deeb, R.A. Mahmoud, $\beta$-open sets and $\beta$-continuous mapping, Bull. Fac. Sci. Assiut Univ.A, 12 (1983), 77-90.

[2]. Radhi I. M. Ali, Jalal H. Hussein and Suhad K. Hameed, On Semi-Pre Irresolute Topological Vector Spaces, Diyala Journal for Pure Sciences, Vol. 14, No.3, (July 2018), 183 191.

[3]. Talal Al-Hawary, $\alpha$-Topological Vector Spaces and $\alpha$-irresolute gauges, Boletin de Matematicas 24(2), (2017), 149 - 154.

[4]. Talal Al-Hawary, $\alpha$-Topological Vector Spaces, Hacettepe Journal of Mathematics and Statistics, Vol. 47, No. 5, (2018), 1102 - 1107.

[5]. D. Andrijevic, Semi - preopen sets, Mat. Vesnik, 38 (1986), $24-32$.

[6]. C.K. Basu and M.K. Ghosh, $\beta$-Closed spaces and $\beta-\theta-$ subclosed graphs European Journal of Pure and Applied Mathematics, Vol. 1, No. 3, (2008), $40-50$.

[7]. G. Aslim, A. Guler Caksu and T. Noiri, On $\pi \mathrm{gs}-$ Closed sets in topological spaces. Acta Mathematica Hungarica 112 (2006), 275-283.

[8]. M. Caldas, Functions with strongly $\beta-\theta$-Closed graphs , J. Adv. Studies Topology, 3 (2012), $1-6$. 
[9]. M. Caldas, On contra $\beta \theta$-continuous functions, Proyecciones Journal Math. 39(4) (2013), 333 - 342.

[10]. Miguel Caldas, Other Characterizations of $\beta-\theta-\mathrm{R}_{0}$ Topological Spaces, Tamkang Journal of Mathematics, Topological Spaces, Volume 44, Number 3, (2013), $303-311$.

[11]. A. Devika and A. Thilagavathi, $\mathrm{M}^{*}$-open Sets in Topological Spaces, International Journal of Mathematics and Applications, Volume 4, Issue 1 - B (2016), pp. 1 - 8 .

[12]. A. Devika and R. Vani, On $\pi \mathrm{g}^{*} \beta$-Closed Sets in Topological Spaces, Journal of Applied and Computational Mathematics, Volume 7, Issue 3 (2018), 1 - 5.

[13]. J. Dontchev and T. Noiri, On generalizing semi-preopen sets, Mem Fac Sci Kochi Univ Ser A (Math) 16 (1995), $35-48$.

[14]. A.I. El-Maghrabi and M.A. Al-Johany, MOpen Sets in Topological Spaces, Poineer Journal of Mathematics and Mathematical Sciences, Volume 4, Number 2 (2011), pp. 213 - 230.

[15]. Hariwan Zikri Ibrahim, $\alpha$-topological vector spaces, Science Journal of University of Zakho, Vol. 5, No. 1(2017) pp. 107 - 111.

[16]. J. Horvath, Topological vector spaces and distributions, Addison and Wesley Publishing Comp., 1966, London.

[17]. M. Khan and B. Ahmad, On P-Regular Spaces, Math. Today, XIII, (1995), $51-56$.

[18]. M. Khan, T. Noiri and M. Hussain, On $\mathrm{s}^{*} \mathrm{~g}$-closed and $\mathrm{s}^{*}$-normal spaces, Journal of Natural Sciences and Mathematics, CODEN, JNSMAC (April \& October 2008) 48, pp. 31 41.

[19]. Moiz ud Din Khan, S. Azam and M. S. Bosan, s-topological vector spaces, Journal of Linear and Topological Algebra. 4(2) (2015), 153 $-158$.

[20]. M. Khan and M. I. Iqbal, On irresolute topological vector spaces, Advances in Pure Mathematics 6 (2016), $105-112$.

[21]. A. Kolomogroff, Zur Normierbarkeit eines topologichen linearem Raumes, Studia Mathematica, 5 (1934), 29 - 33.

[22]. Raja M. Latif, $\omega b$-Topological Vector Spaces, WSEAS Transactions on Mathematics, Volume 119, 2020, Art. \#12, pp. 119 -132.

[23]. N. Levine, Semi-open sets and Semi-continuity in topological spaces, Amer. Math. Monthly. 70 (1963), $36-41$.
[24]. N. Levine, Generalized closed sets in topological spaces, Rend. Circ. Mat. Palermo,vol.19(2),(1970), 89-96.

[25]. Sabiha I. Mahmood and Jumana S. Tareq, On $\mathrm{s}^{*} \mathrm{~g}-\alpha-$-Open Sets in Topological Spaces, Ibn Al-Haitham Journal for Pure and Applied Sciences, Vol. 27 (3), (2014), 542 - 555.

[26]. T. Noiri, Weak and strong forms of $\beta$-irresolute functions, Acta Math. Hungar. 99 (2003), $315-328$

[27]. O. Njastad, On some classes of nearly open sets, Pacific Journal of Mathematics 15 (1965), $961-970$.

[28]. Madu Ram and Shallu Sharma, Corrigendum to: On Irresolute Topological Vector Spaces, NTMSCI 6, N. 4 (2018), 40 - 44.

[29]. JH Park and JK Park, On $\pi \mathrm{gp}$ - continuous functions in topological spaces, Chaos Solitons \& Fratals 20 (2004), $467-477$.

[30]. Madhu Ram, Shallu Sharma, Sahil Billawria and Amjad Hussain, On almost $\mathrm{s}$-topological vector spaces, Journal of Advanced Studies in Topology 9(2) (2018), 139 146.

[31]. Thanjavur N. Rajesh, Tiruchirappalli V. Vijayabhathi, On Strongly Preirresolute Topological Vector Spaces, Mathematica Bohemica, 138 (1), (2013), 37 - 42.

[32]. A. Robert and S. Pious Missier, A New Class of Nearly Open Sets, International Journal of Mathematical Archive, 3(7), 2012, 1 - 8 .

[33]. A. Robert and S. Pious Missier, A New Class of Sets Weaker than Alpha-open Sets, International Journal of Mathematics and Soft Computing, 4(2) (2014), $197-206$.

[34]. A. Robert and S. Pious Missier, Between Alpha-closed Sets and Semi Alpha-closed Sets, International Journal of Modern Engineering Research, 4(6) 2014, 34 - 41.

[35]. A. Robert and S. Pious Missier, Semi-Star-Alpha-Open Sets and Associated Functions, International Journal of Computer Applications, Volume 104, No 16 (October 2014), $24-29$.

[36]. Shallu Sharma, Madhu Ram and Sahil Billawria, On Almost Pretotopological Vector Spaces, Open Access Library Journal, Volume 5, (2018), $1-10$.

[37]. S. Sharma, M. Ram, On $\beta$-topological vector spaces, Journal of Linear and Topological Algebra, Vol. 08, No. 01, (2019), 63 - 70.

[38]. Shallu Sharma, Sahil Billawria, Madhu Ram, Tsering Landol, On Almost 
$\beta$-topological Vector Spaces, Open Access Library Journal, Volume 6, (2019), 1 - 9.

[39]. N. V. Velicko, H-closed topological spaces, Amer. Math. Soc. Transl., 78 (1968), 103 $-118$.

[40]. Stephen Willard and Raja M. Latif, Semi-Open Sets and Regularly Closed Sets in Compact Metric Spaces, Mathematica Japonica, Vol. 46, No.1, (1997), $157-161$.

[41]. S. Willard, General Topology, Addison Wesley Publishing Co., Reading, Mass. - London - Don Mills, Ont., 1970.
Creative Commons Attribution License 4.0 (Attribution 4.0 International, CC BY 4.0)

This article is published under the terms of the Creative Commons Attribution License 4.0

https://creativecommons.org/licenses/by/4.0/deed.en_US 